Localizador: 19037 doi: $10.35366 / 93976$

\title{
Sífilis secundaria, 590 dilemas resueltos
}

\author{
Secondary syphilis, 590 dilemmas resolved \\ Alcira Bermejo, ${ }^{*}$ Viviana Leiro, ${ }^{*}$ Malena Colasanti, ${ }^{\ddagger}$ Mercedes Solís Ramírez, ${ }^{\ddagger}$ \\ Mariel McGuire, ${ }^{\S}$ Liliana Olivares"
}

Palabras clave:

Sifilis secundaria, exantema, adenopatías.

Keywords: Secondary syphilis, exantema, adenopathies.
* Médica de planta del Sector ETS.

Médica concurrente.

${ }^{\S}$ Médica Dermatóloga.

| Jefa de Servicio.

Hospital de Infecciosas "Dr. Francisco Javier Muñiz», CABA, Argentina

Recibido:

22/Julio/2019.

Aceptado:

23/Octubre/2019.

\section{RESUMEN}

Introducción: La Organización Mundial de la Salud (OMS hasta agosto de 2016 reportó que cada año 5.6 millones de personas contraen sífilis en todo el mundo. En la actualidad, existe un incremento global de la incidencia de la enfermedad, que es constante en la última década. La sífilis secundaria es la etapa de la enfermedad conocida como «la gran simuladora», debido al polimorfismo que muestra en sus diversas formas de presentación, lo que suele dificultar el diagnóstico con el agravante de la infectividad que representa este estadio de la enfermedad. Objetivos: Mostrar las formas actuales de presentación de la sífilis secundaria de acuerdo con lo observado en la sección de Enfermedades de Transmisión Sexual (ETS) en un hospital de enfermedades infecciosas en la Ciudad Autónoma de Buenos Aires (CABA), a la luz del aumento creciente de casos de la enfermedad. Observar el diverso espectro de presentación y establecer diferentes parámetros identificatorios de la enfermedad que puedan orientar por su sola presencia a la sífilis secundaria. Material y métodos: Se realizó estudio transversal de los casos de sífilis diagnosticados entre marzo de 2014 y marzo de 2017 en el sector de ETS. Los pacientes fueron evaluados según edad, género, sexualidad y formas clínicas de presentación. Resultados: Se diagnosticaron 1,236 casos de sífilis temprana. La prevalencia de sífilis secundaria fue de $48 \%$. Setenta por ciento de los afectados eran hombres. Cuarenta y nueve por ciento de los casos de ambos sexos se presentaron en la franja de entre 20 y 30 años. El $68 \%$ de los enfermos refirió ser heterosexual. El $44 \%$ de los afectados tuvo compromiso cutáneo mucoso, 28\% sólo mostró compromiso de mucosas y 47\% presentó adenopatías periféricas. Conclusión: Esta numerosa cohorte, la mayor de acuerdo con la bibliografía publicada a la fecha, muestra una incidencia sostenida de sífilis secundaria en un hospital público. Se destaca que ante la aparición de exantema con o sin compromiso mucoso y adenopatías se debe pensar hoy más que nunca en sífilis secundaria entre los diagnósticos diferenciales.

\section{ABSTRACT}

Introduction: As reported by the World Health Organization (WHO), until August 2016, 5.6 million people were infected with syphilis every year. Currently, there is a global increase in the incidence of the disease, which is constant in the last decade. Secondary syphilis is the stage of the disease known as «the great imitatory, due to the polymorphism that it shows in its various forms of presentation, which usually makes diagnosis difficult with the aggravating factor of the infectivity that represents this stage of the disease. Objectives: To show the current forms of presentation of the secondary syphilis according to what was observed in the Sexually Transmitted Diseases (STDs) section in a hospital of infectious diseases in the Autonomous City of Buenos Aires (CABA), in light of the increasing number of cases of the disease. To observe the diverse spectrum of presentation and to establish different identifying parameters of the disease which can orient, by its simply presence, to the identification of secondary syphilis. Material and methods: a cross-sectional study of the cases of syphilis diagnosed between March 2014 and March 2017 in the STD section was carried out. The patients were evaluated according to age, gender, sexuality and clinical forms of presentation. Results: 1,236 cases of early syphilis were diagnosed. Prevalence of secondary syphilis was $48 \%$. 70\% were men, $49 \%$ of both genders were between 20 and 30 years old. Sixty-eight percent (68\%) of the patients reported to be heterosexual. Forty-four percent (44\%) had mucosal and cutaneous manifestations, while $20 \%$ showed only mucosal manifestations and $47 \%$ had peripheral adenopathies. Conclusion: This large cohort, the largest according to the bibliography published to date, shows a sustained incidence of secondary syphilis in patients of a public hospital. It is important to emphasize that in the presence of exanthema, with or without mucosal compromise and adenopathy, secondary syphilis should be considered as part of the differential diagnosis.

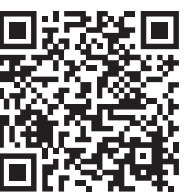

\section{INTRODUCCIÓN}

L a Organización Mundial de la Salud (OMS) hasta agosto de 2016 reportó que cada año 5.6 millones de personas contraen sífilis en todo el mundo. En la actualidad, existe un incremento global de la incidencia de la enfermedad, que es constante en la última década. ${ }^{1}$ 
La sífilis adquirida temprana es la etapa más importante desde el punto de vista epidemiológico porque es la que más contagia y se extiende desde el momento de adquisición de la enfermedad hasta el primer año; incluye la sífilis primaria, la sífilis secundaria y la sífilis serológica temprana. Se calcula que por cada caso índice hay 10 pacientes más con sífilis que no consultan o son asintomáticos, por lo que la intervención médica para cortar la cadena epidemiológica con un diagnóstico y tratamiento precoz es fundamental. ${ }^{2}$

La sífilis secundaria es la etapa de la enfermedad conocida como «la gran simuladora», debido al polimorfismo que muestra en sus diversas formas de presentación, lo que suele dificultar el diagnóstico con el agravante de la infectividad que representa este estadio de la enfermedad. ${ }^{3}$

La sífilis es un problema actual de salud pública, principalmente por su repercusión en mujeres embarazadas, neonatos y pacientes con serología reactiva para $\mathrm{VIH}$.

Deben centrarse esfuerzos en la educación de la población para una sexualidad responsable, en la detección precoz del primer estadio y en la profilaxis temprana de los contactos sexuales aún asintomáticos. ${ }^{4}$

Se realizó un estudio clínico-epidemiológico en el sector de ETS del Hospital Muñiz.

Objetivos. Mostrar las formas actuales de presentación de la sífilis secundaria de acuerdo con lo observado en el sector de ETS en un hospital de enfermedades infecciosas en CABA, a la luz del aumento creciente de casos de la enfermedad.

Observar el diverso espectro de presentación y establecer diferentes parámetros identificatorios de la enfermedad que puedan orientar por su sola presencia a la sífilis secundaria.

\section{MATERIAL Y MÉTODOS}

Se realizó un estudio observacional prospectivo, de marzo de 2014 a marzo de 2017, donde se registraron en una base de datos los casos de sífilis diagnosticados en la sección de ETS, del Servicio de Dermatología del Hospital de Infecciosas «Dr. Francisco Javier Muñiz», de la Ciudad de Buenos Aires. Se registraron todos los datos epidemiológicos de los pacientes (edad, sexo y orientación sexual) y sus características clínicas (formas de presentación: cutánea, mucosa, cutaneomucosa y adenopatías con sus variantes). Se calcularon sus respectivos índices de confianza del 95\%, considerando como variables dependientes las formas clínicas y como variables independientes los datos epidemiológicos. Criterios de inclusión: pacientes con diagnóstico de sífilis secundaria ambulatorio, que concurrieron por primera vez al sector de ETS por la patología en cuestión. El diagnóstico fue realizado a partir de las manifestaciones clínicas, el fondo oscuro y la prueba de VDRL (Venereal Disease Research Laboratory) cuantitativa.

Criterios de exclusión: pacientes con diagnóstico de sífilis secundaria con compromiso neurológico.

Criterios de eliminación: pacientes con diagnóstico de sífilis secundaria cuyas historias clínicas no se encontraron o tenían datos incompletos.

\section{RESULTADOS}

En el lapso analizado se realizaron 3,942 consultas de primera vez en la sección de ETS, de las que 1,236 fueron sífilis temprana, y de ellas 590 sífilis secundaria. La incidencia de la sífilis secundaria sobre la cantidad de consultas de primera vez por año fue en 2014 de 183/1,163, que representó 15\%; en 2015 fueron 233/1,418 (16\%) y en 2016 174/1,361 (13\%).

La prevalencia de la sífilis secundaria sobre el total de sífilis temprana en nuestro servicio fue de $47.73 \%$, con una relación de una sífilis secundaria por cada dos sífilis tempranas. Es preciso señalar que todos los resultados siguientes fueron calculados con un índice de confianza (IC) del 95\%. De los 590 pacientes con diagnóstico de sífilis secundaria, 70.5\% (416 pacientes, $66-74 \%)$ fueron hombres y $29.5 \%$ (174, $25-33 \%)$ fueron mujeres, con una edad media de presentación de 29 años (28-30\%), con un máximo de 86 años y un mínimo de 14 años. En cuanto a la orientación sexual, $68 \%(404,64-72 \%)$ refirieron ser heterosexuales, $26 \%$ (154, 23-29\%) hombres que tienen sexo con hombres $(\mathrm{HSH}), 3 \%(17,2-4 \%)$ bisexuales y $2 \%(15,1-3 \%)$ transexuales.

Según las formas de presentación clínicas se armaron cuatro modelos: cutáneo, mucoso, cutaneomucoso y sólo compromiso de ganglios. El compromiso cutáneo representó $17 \%$ (100 pacientes, $14-20 \%$ ) donde la mitad tuvo adenopatías (50, 46-60\%). Dentro de las variedades clínicas, de los que presentaron exantemas, 50 pacientes tuvieron la forma papular o morbiliforme, uno psoriasiforme, uno liquenoide y uno varioliforme. La edad media de presentación fue de 29 años (28-30\%). Sesenta y siete por ciento (67 pacientes, $57-75 \%$ ) fueron hombres y $33 \%(33,25-43 \%)$ mujeres. Con respecto a la orientación sexual, $68 \%(68,64-72 \%)$ refirió ser heterosexual, $26 \%$ $(26,22-30 \%) \mathrm{HSH}, 3 \%$ (tres, $2-5 \%$ ) bisexual y $3 \%$ (tres, $1-4 \%)$ transexual. 
La forma mucosa se presentó en 30\% $(178,26-34 \%)$ del total de las sífilis secundarias, de las cuales 56\% (99, 48$63 \%)$ presentó adenopatías. De estos pacientes, 71 tuvieron compromiso oral, 59 genital (12 con chancros persistentes) y 53 ambos (oral y genital). La edad media de presentación fue de 28 años (26-29\%). En cuanto al género hubo 67\% $(118,59-73 \%)$ hombres y $33 \%(59,27-41 \%)$ mujeres. Sobre la orientación sexual, $70 \%(124,63-76 \%)$ refirieron ser heterosexuales, $27 \%$ (48, 21-34\%) HSH, 0.6\% (uno, 0.1-3\%) bisexual y $2 \%$ (cuatro, $1-6 \%$ ) transexual.

La forma cutaneomucosa se observó en 48\% (284, 44$52 \%)$ de los pacientes con sífilis secundaria. Se acompañó de adenopatías en $71 \%(202,75-76 \%)$ de los pacientes. Las formas clínicas del exantema que presentaron fueron 154 sujetos con afectación de tipo papular o morbiliforme, cinco psoriasiforme, dos liquenoide, tres urticariforme y uno varioliforme; y como variantes infrecuentes tres tuvieron lesiones específicas interdigitales en pies y uno compromiso umbilical. Se evidenció afectación de mucosa oral en 89 pacientes, genital en 93 (32 con chancros persistentes) y ambas en 97 sujetos. La edad media de presentación fue de 30 años (29-32\%). De ellos 74\% (210, $68-79 \%)$ fueron hombres y $26 \%(74,21-31 \%$, ) mujeres. La orientación sexual fue $67 \%(192,62-73 \%)$ heterosexual, $26 \%(35,22-32 \%) \mathrm{HSH}, 4 \%(11,2-7 \%)$ bisexual y $2 \%$ (seis, $1-4 \%)$ transexuales.

Por último, el compromiso exclusivo de ganglios se evidenció en 5\% (27 pacientes, 3-7\%) del total de los sujetos con sífilis secundaria. La edad media de presentación fue de 26 años (25-38\%). Setenta y ocho por ciento (21, $52-92 \%$ ) fueron hombres y $22 \%$ (seis, $8-48 \%$ ) mujeres. Sobre la orientación sexual, $74 \%(20,48-90 \%)$ refirió ser heterosexual, 19\% (cinco, 6-45\%) HSH, 7\% (dos, 1-27\%) bisexual y ninguno transexual.

\section{DISCUSIÓN}

La sífilis es una enfermedad infectocontagiosa causada por el Treponema pallidum subespecie pallidum. Presenta afectación sistémica y es de evolución crónica, con periodos de exacerbación e intervalos de latencia. ${ }^{5}$

El nombre sífilis fue acuñado por primera vez en 1530 por el médico italiano Fracastoro. Años más tarde, Jean Fennel introduce el término Lúes (plaga en latín), nombre con el cual también se conoce a la enfermedad. ${ }^{2,5}$

Los avances más importantes fueron reportados en el siglo XX. En 1905, el zoólogo Fritz Schaudinn y el dermatólogo Erich Hoffmann descubrieron el agente causal, el cual fue cultivado en 1911 por Noguchi y aislado en el sistema nervioso central de un tabético en el año 1913. En 1906 se desarrollaron por primera vez las reacciones serológicas para sífilis por Wassermann, Neisser y Bruck, y en 1928 Fleming descubre la penicilina, la cual es usada como tratamiento definitivo de la sífilis en 1943, reportándose el primer caso tratado en Argentina en 1947. En 1941 comienza el uso de las pruebas no treponémicas para diagnóstico y en 1962 la FTA-abs (Fluorescent Treponemal Antibody-Absorption) como prueba específica. En la década de los 90, inician los estudios con PCR (Polymerase chain reaction), y hacia fines del siglo XX se logra la secuenciación completa del genoma del Treponema pallidum. ${ }^{4-7}$

La prevalencia de la sífilis ha variado a lo largo de la historia. Durante los siglos XV y XVI, alrededor de 15\% de la población europea la padecía y ocasionaba miles de muertes. ${ }^{7}$ La OMS, hasta agosto de 2016, reportó que cada año 5.6 millones de personas contraen sífilis en todo el mundo, ${ }^{1}$ lo cual resulta paradójico, ya que en la actualidad se sabe que una conducta sexual segura y responsable puede prevenir su contagio, lo que hace más notable aún que en pleno siglo XXI estas enfermedades lejos de disminuir se encuentran en ascenso.

No resulta fácil caracterizar con precisión y exactitud la situación epidemiológica de esta enfermedad. Tanto los sistemas de vigilancia y notificación de casos como las coberturas que alcanzan los programas y los recursos disponibles difieren de un país a otro. ${ }^{8}$ En nuestro Servicio de Dermatología en el sector de ETS se registró en el periodo analizado un total de 1,236 casos de sífilis temprana, siendo la prevalencia de sífilis secundaria cercana a 50\%. Si lo comparamos con lo registrado en el mismo servicio durante un periodo de 10 años (1998-2008), donde se registraron 1,541 casos de sífilis adquirida temprana (menos de un año de enfermedad), ${ }^{3}$ podemos afirmar que hemos sido testigos de un fenomenal aumento de nuevos casos. De la misma forma, en los años 2001 a 2005 la prevalencia de sífilis aumentó cada año hasta alcanzar cifras de 2.97 por 100,000 habitantes en Estados Unidos. ${ }^{2}$

La enfermedad se puede adquirir por contacto sexual, de forma congénita a través de la placenta y con menos frecuencia por transfusión de sangre humana contaminada o inoculación accidental directa. ${ }^{5,9}$

La proporción entre hombres y mujeres varía según la situación geográfica. ${ }^{10}$ Según el Centers for Disease Control and Prevention (CDC), durante el periodo 2000-2015, el aumento de la tasa de sífilis primaria y secundaria se debió principalmente al aumento de casos entre hombres. Sin embargo, durante 2013-2015, la tasa aumentó tanto en hombres como en mujeres. Durante 2014-2015, las tasas 
se incrementaron $18.1 \%$ entre los hombres y $27.3 \%$ entre las mujeres. ${ }^{11} \mathrm{Al}$ igual que se describe en la bibliografía, la enfermedad es más frecuente en hombres, ${ }^{1,5,10}$ datos que coinciden con los obtenidos en nuestro estudio.

Si bien el CDC en el año 2015 publicó que los HSH siguen representando la mayoría de los casos de sífilis en Estados Unidos, ${ }^{11}$ en nuestra casuística sólo $26 \%$ fueron $\mathrm{HSH}$, mientras que más de la mitad de los pacientes eran heterosexuales. Hallazgos semejantes a los nuestros fueron encontrados en un hospital público (ciudad de La Plata. Provincia de Buenos Aires) por Gareis en un estudio, donde el mayor porcentaje de los pacientes eran heterosexuales (años 1999-2003). ${ }^{5}$

La evolución natural de la sífilis se divide en varias etapas sucesivas: sífilis primaria, sífilis secundaria, sífilis latente y sífilis tardía o terciaria. Cada estadio de la enfer-

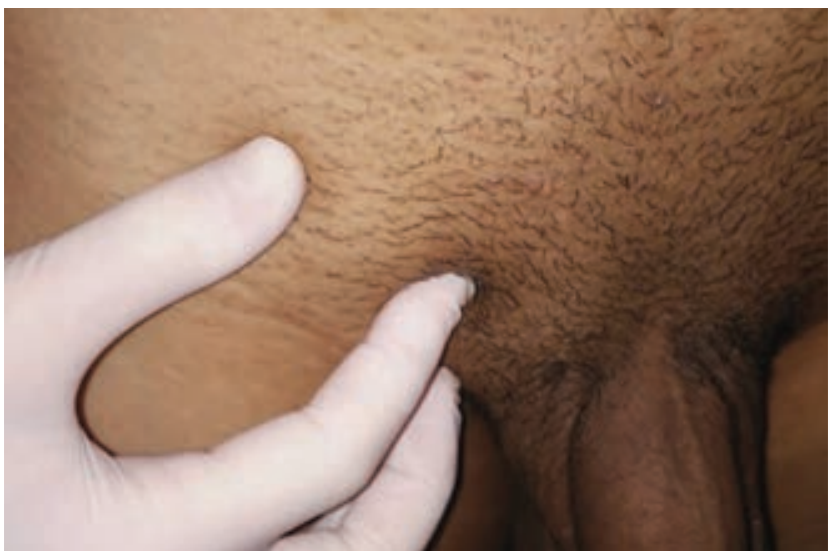

Figura 1: Adenopatía inguinal.
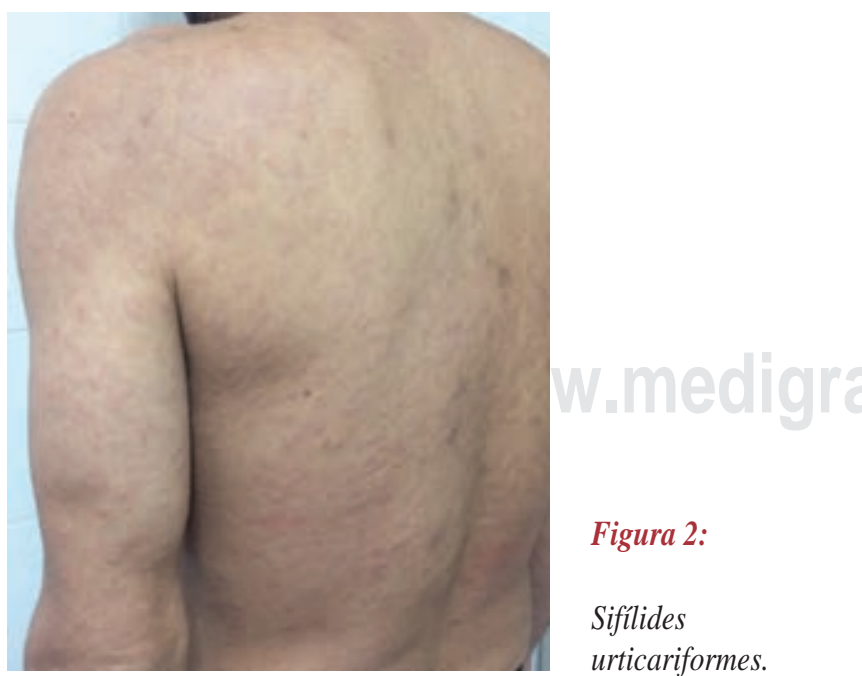

Figura 2:

Sifílides urticariformes.

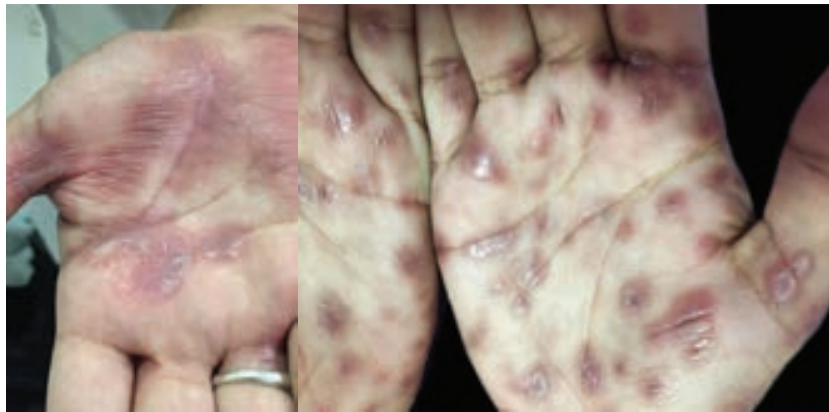

Figura 3: Sifílides palmares.

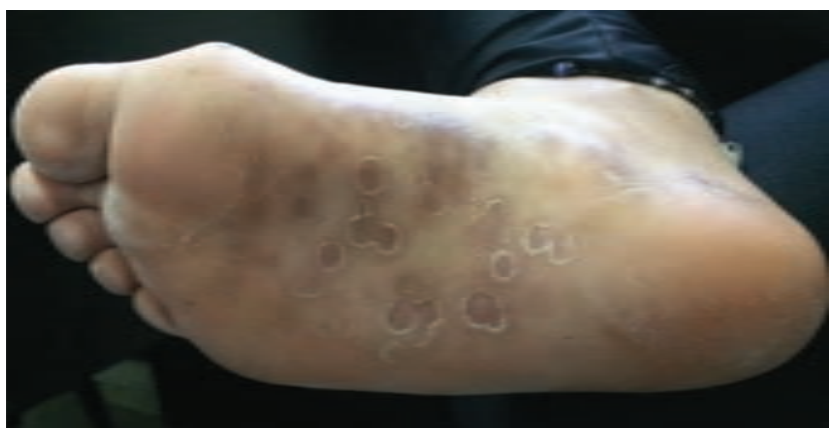

Figura 4: Sifílides plantares.

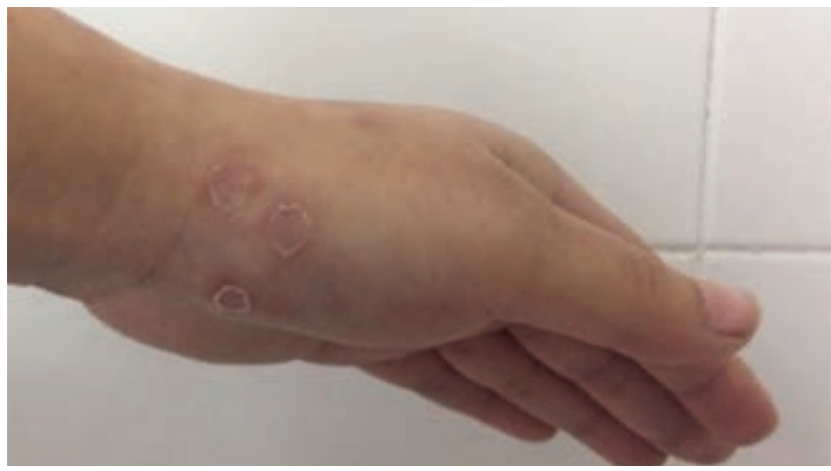

Figura 5: Collarete de Biett.

medad presenta manifestaciones clínicas, inmunológicas, serológicas y anatomopatológicas distintivas. ${ }^{5,12}$

La sífilis primaria aparece tras un periodo de incubación de 10 a 90 días (promedio de tres semanas) y está representada clínicamente por el chancro de inoculación y las adenopatías satélites o regionales. ${ }^{5,9}$ En los individuos no tratados, los treponemas proliferan en el chancro y son transportados por vía linfática al torrente sanguíneo, desde donde se diseminan por todo el cuerpo (sepsis treponémi- 
ca), dando inicio al periodo secundario, ${ }^{13}$ el estadio clínico más florido de la infección. El tiempo en el que las lesiones secundarias aparecen básicamente depende de dos factores: la virulencia del treponema y la respuesta sistémica del huésped. ${ }^{3,13}$ Los treponemas invaden todos los órganos y

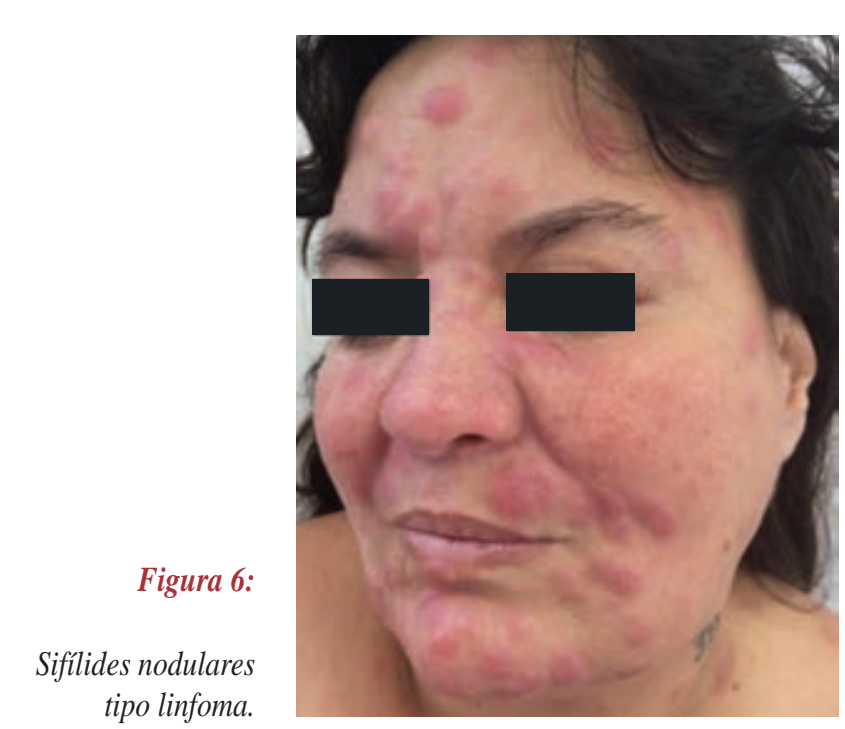

\begin{tabular}{|c|c|c|c|c|}
\hline & $\begin{array}{l}\text { Cutáneo } \\
\text { (n) }\end{array}$ & $\begin{array}{l}\text { Cutaneomucosa } \\
\text { (n) }\end{array}$ & $\begin{array}{l}\text { Mucosa } \\
\text { (n) }\end{array}$ & $\begin{array}{c}\text { Sólo } \\
\text { adenopatías } \\
\text { (n) }\end{array}$ \\
\hline \multicolumn{5}{|l|}{ Sexo } \\
\hline Total & 100 & 284 & 177 & 28 \\
\hline Masculino & 67 & 210 & 118 & 21 \\
\hline Femenino & 33 & 74 & 59 & 7 \\
\hline \multicolumn{5}{|l|}{ Edad } \\
\hline Media & 29 años & 30 años & 28 años & 26 años \\
\hline \multicolumn{5}{|l|}{ Orientación sexual } \\
\hline Heterosexuales & 67 & 192 & 124 & 20 \\
\hline Homosexuales & 33 & 35 & 48 & 5 \\
\hline Bisexuales & 3 & 11 & 1 & 2 \\
\hline Transexuales & 3 & 6 & 4 & \\
\hline \multicolumn{5}{|l|}{ Exantema } \\
\hline Papular & 50 & 154 & & \\
\hline Psoriasiforme & 1 & 5 & & \\
\hline Varioliforme & 1 & 1 & & \\
\hline Liquenoide & 1 & 2 & & \\
\hline Urticariforme & 0 & 3 & & \\
\hline $\begin{array}{l}\text { Presencia de } \\
\text { adenopatías }\end{array}$ & 50 & 202 & 99 & \\
\hline \multicolumn{5}{|l|}{ Mucosas } \\
\hline Sólo oral & & 89 & 71 & \\
\hline Sólo genital & & 93 & 59 & \\
\hline Ambas & & 97 & 53 & \\
\hline Chancro persistente & & 32 & 12 & \\
\hline
\end{tabular}

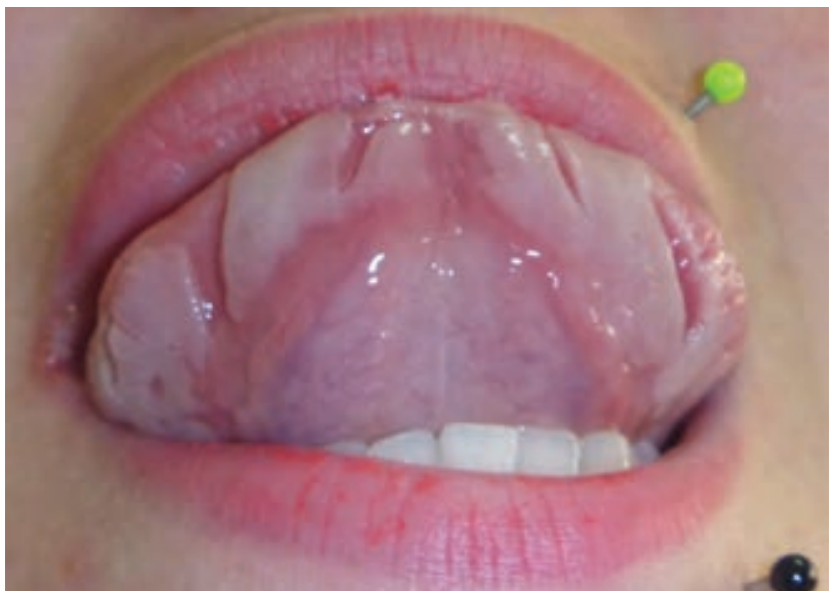

Figura 7: Sifílides opalinas.

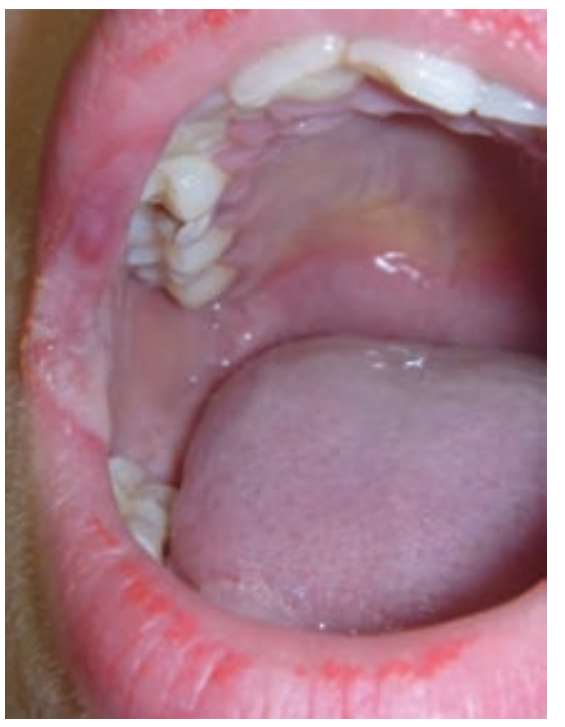

Figura 8:

Queilitis angular.

la mayoría de los líquidos orgánicos. Las manifestaciones son muy variadas, por lo que se le ha adjudicado a la enfermedad el nombre de «la gran simuladora». ${ }^{5,9,13}$ La sífilis secundaria no tratada se resuelve de manera espontánea de tres a 12 semanas, dejando al paciente libre de lesiones y síntomas, esto correspondiente al periodo de latencia, momento en el cual la enfermedad sólo se evidencia a través de las pruebas serológicas. ${ }^{12,13}$ Años más tarde sobreviene el periodo terciario, hoy en día infrecuente. ${ }^{5}$ Cabe destacar que el T. pallidum puede invadir el sistema nervioso central (SNC) en cualquier fase de la enfermedad. ${ }^{14}$

Clínicamente la sífilis secundaria puede acompañarse de síntomas y signos inespecíficos como febrícula, ma- 
lestar, cefalea, odinofagia, artralgias, conjuntivitis y hepatoesplenomegalia en $12 \%$ de los pacientes. ${ }^{2,12}$ Se puede desarrollar uveítis, que suele ser unilateral en $56 \% .^{2}$ Las adenopatías generalizadas indoloras están presentes entre 70 y $85 \%$ de los casos (Figura 1). ${ }^{2,5}$ En nuestro estudio un alto porcentaje de pacientes presentó adenopatías (351 casos). El compromiso exclusivo de ganglios se evidenció en $5 \%$ (27 casos). En piel las lesiones son polimorfas y se denominan sif́lides, representan reacciones inflamatorias de los tejidos en respuesta a la acumulación metastásica de treponemas. ${ }^{3,5,13,15}$ De forma inicial aparece un exantema macular evanescente asintomático que a menudo se pasa por alto (roséola sifilítica). Días más tarde,

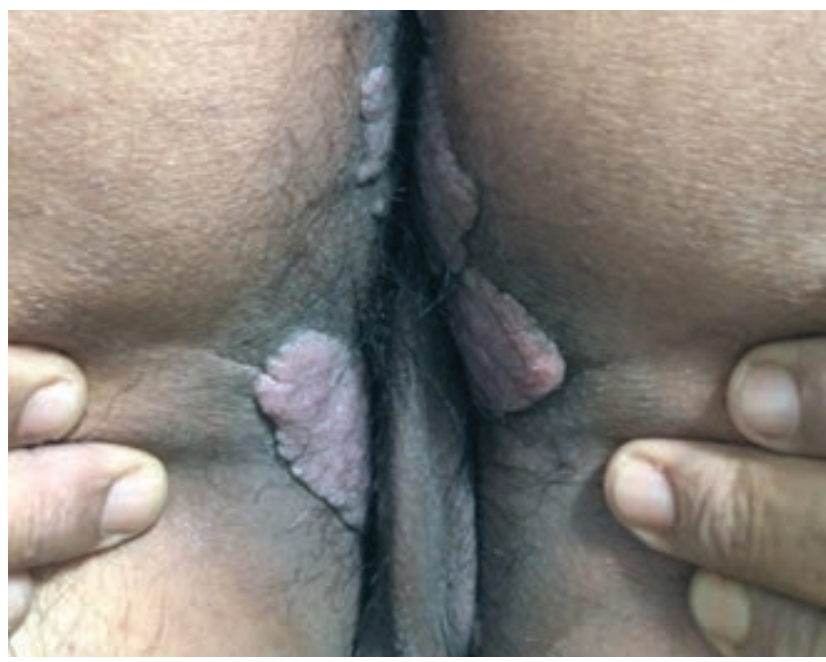

Figura 9: Condilomas planos perianales.

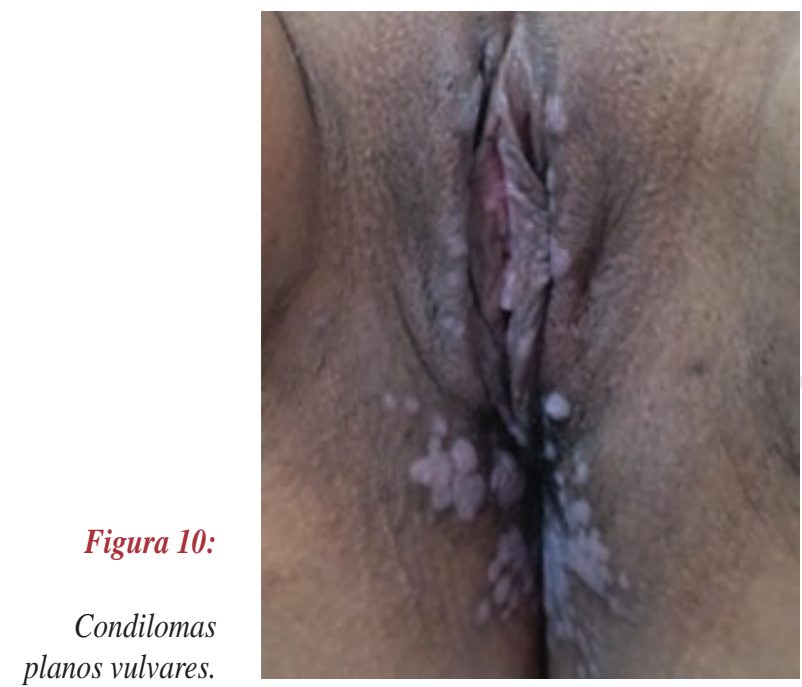

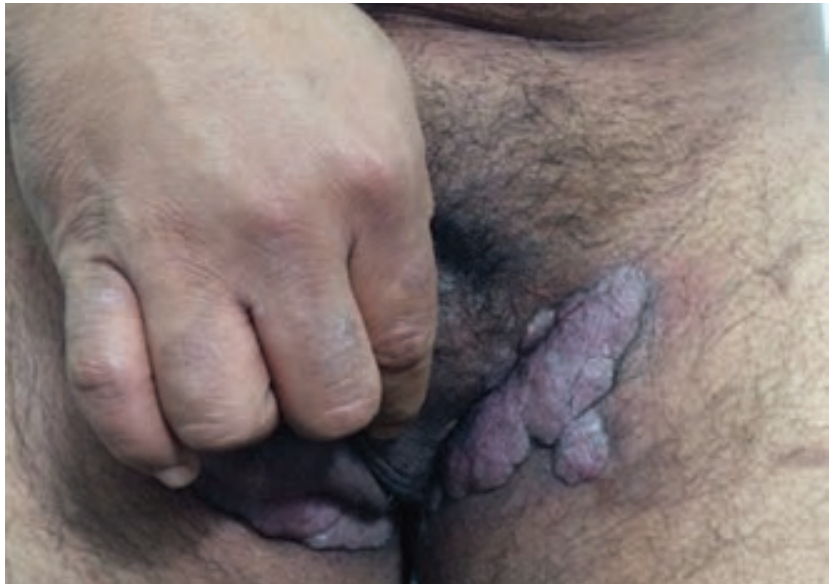

Figura 11: Condilomas planos inguinoescrotales.

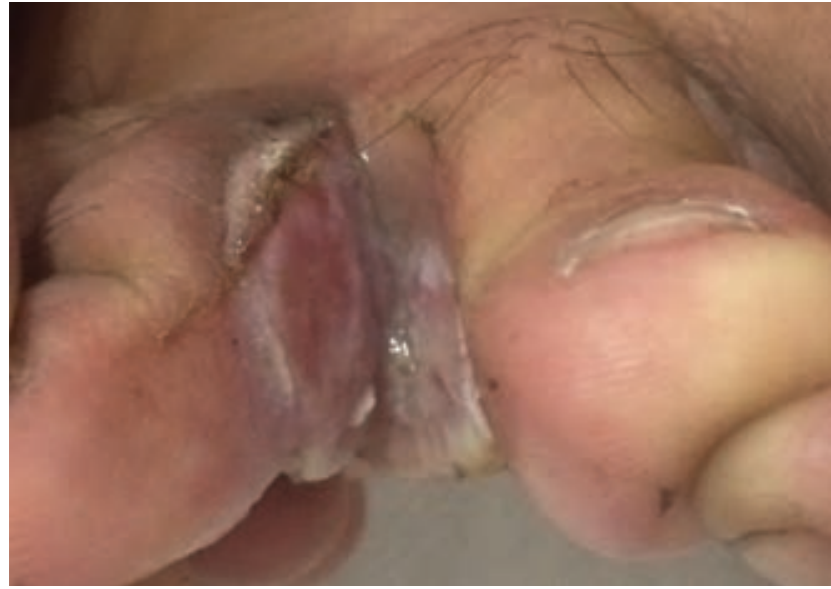

Figura 12: Condiloma plano interdigital.

aparece una erupción papuloescamosa, simétrica, que compromete tronco y extremidades incluyendo palmas y plantas (Figuras 2 a 4). Suelen ser lesiones eritematoescamosas con descamación periférica «en collarete» (Figura 5). ${ }^{1,13,16}$ Generalmente no pruriginosas, aunque existen reportes de que este síntoma puede estar presente hasta en $40 \%$ de los casos. ${ }^{10,16}$ En ocasiones el chancro puede persistir y cabalgar con lesiones de secundarismo como observamos en 44 de los 590 pacientes. ${ }^{3,10}$ Veintinueve punto seis por ciento de las manifestaciones cutáneas de la sífilis muestran una morfología atípica: liquenoides, micropapulares, foliculares, vesiculares, corimbiformes, psoriasiformes, nodular, anular, frambuesiforme, nóduloulcerativa, papuloescamosa fotodistribuida, tipo linfoma y pustulosa (Figura 6). 2,12,17 Estas manifestaciones suelen 
retardar el diagnóstico a menos que se acompañen de lesiones típicas. ${ }^{16}$ Las lesiones cutáneas curan sin cicatriz aunque pueden dejar cambios pigmentarios (sifílides pigmentarias)..$^{10}$ En nuestra casuística encontramos que $17 \%$ de los pacientes presentaba lesiones sólo a nivel cutáneo, siendo la manifestación más frecuente el exantema papular, seguido de la forma psoriasiforme (Tabla 1). Se destaca que la mayoría de los pacientes (48\%) presentaron lesiones tanto a nivel cutáneo como mucoso (forma cutaneomucosa). El compromiso mucoso es muy frecuente y característico de sífilis secundaria con lesiones altamente contagiosas. ${ }^{13}$ En la mucosa oral, pueden presentarse placas blanquecinas redondeadas asintomáticas (sifílides opalinas), que a nivel de lengua dejan áreas depapiladas,

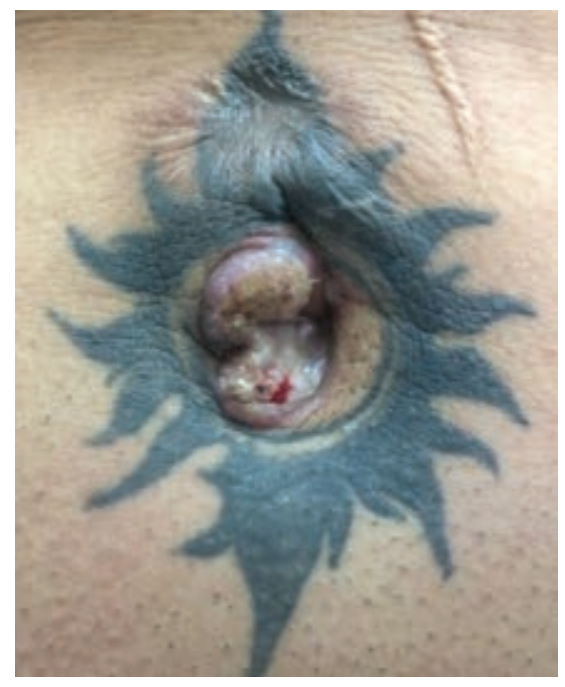

Figura 13:

Condiloma plano umbilical.

Figura 14:

Alopecia de cuero cabelludo y cejas.

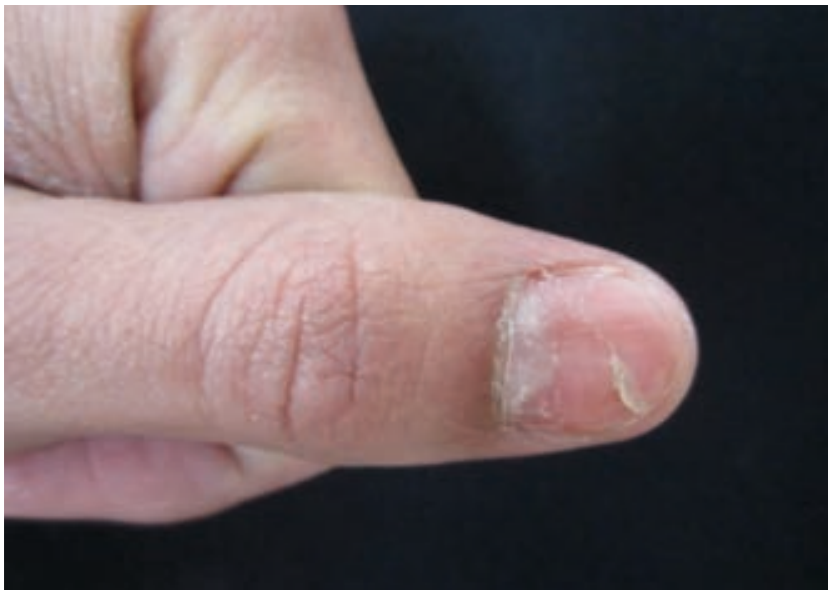

Figura 15: Onicólisis.

lo que se denomina «lengua en pradera segada». Se puede observar además enantema faucial y el compromiso de las comisuras labiales dando una queilitis que característicamente compromete piel, mucosa y semimucosa labial (Figuras 7 y 8). ${ }^{10}$ En nuestro estudio $30 \%$ de los pacientes presentaron sólo lesiones a nivel de mucosas, de los cuales la mayoría de ellos tenían compromiso tanto a nivel de mucosa oral como genital. Cuando las sifílides se localizan a nivel de pliegues cutáneos o cutaneomucosos reciben el nombre de condilomas planos. Su localización habitual son los pliegues interglúteo, perianal y la vulva (Figuras 9 a 11). Y con menos frecuencia en pliegues submamarios, axilares, auriculares e interdigitales (Figuras 12 y 13).2,13,15 La afectación de los anexos cutáneos se evidencia hacia el final de este periodo con la aparición de alopecia no cicatrizal en 3-7\% de los casos. ${ }^{2,13,18}$ Puede afectar además cejas, pestañas, tronco y pubis (Figura 14).$^{18}$ También pueden verse comprometidas las uñas y la región periungueal (Figura 15).2

\section{CONCLUSIONES}

Este trabajo constituye la mayor casuística de sífilis secundaria publicada en nuestro país y muestra una incidencia sostenida de casos a lo largo de casi un lustro en un hospital público. De acuerdo a nuestra búsqueda tampoco se ha encontrado una cohorte tan numerosa a nivel mundial.

No se encontró correlación entre variables de edad, género y sexualidad con las diferentes formas clínicas.

Se destaca que ante la aparición de exantema con o sin compromiso mucoso y adenopatías se debe pensar, hoy 
más que nunca, en sífilis secundaria entre los diagnósticos diferenciales.

El sector de ETS del Servicio de Dermatología del Hospital Muñiz tiene atención diaria por demanda espontánea, y es, en la actualidad, el único centro de referencia para la atención de estas patologías.

Creemos que este trabajo constituye un aporte al conocimiento del panorama completo del estado de la sífilis secundaria.

\section{AGRADECIMIENTOS}

Agradecemos la colaboración del Dr. Hugo N. Catalano en la corrección estadística de este trabajo.

Correspondencia:

Alcira Bermejo

E-mail: alcirabermejo@gmail.com

\section{BIBLIOGRAFÍA}

1. Centers for Disease Control and Prevention. Sexually Transmitted Disease Surveillance 2016. Atlanta: US Department of Health and Human Services; 2016.

2. Balagula $Y$, Mattei PL, Wisco OJ, Erdag G, Chien AL. The great imitator revisited: the spectrum of atypical cutaneous manifestations of secondary syphilis. Int J Dermatol. 2014; 53 (12): 1434-1441.

3. Bermejo A, Leiro V. Sífilis. El desafío permanente. Dermatol Argent. 2011; 17: 156-159.

4. Vera I, Fernández-Pardal P, Leiro V, Bermejo A. Chancros sifilíticos en el nuevo milenio: 217 (casos) razones para pensar en sífilis. Dermatol Argent. 2012; 18: 442-451.

5. Gareis MC. Sífilis, aspectos epidemiológicos [trabajo científico]. Universidad Nacional de la Plata. Disponible en: http://www. postgradofcm.edu.ar

6. Leitner R, Körte C, Edo D, Braga M. Historia del tratamiento de la sífilis. Rev Argent Dermatol. 2007; 88: 6-19.

7. Berdasquera-Corcho D, Lazo-Álvarez M, Galindo-Santana B, GalaGonzález A. Sífilis: pasado y presente. Rev Cubana Hig Epidemiol [Internet]. 2004 [citado 16 de septiembre de 2017]; 42 (2). Disponible en: http://scielo.sld.cu/scielo.php?script=sci arttext\&pid=S1561300 32004000200008\&lng=es\&nrm=iso>. ISSN 1561-3003.

8. Galban E, Benzaken A. Situación de la sífilis en 20 países de Latinoamérica y El Caribe: año 2006. J Bras Doenças Sex Transm. 2007; 19: 166-172.
9. Contreras E, Zuluaga S, Ocampo V. Sífilis: la gran simuladora. Infect. 2008; 12: 120-127.

10. Katz K. Sifilis. En: Goldsmith L, Katz S, Gilchrest B, Paller A et al. Fitzpatrick Dermatología en Medicina General. Buenos Aires: Ed. Panamericana; 2014. pp. 2471-2492.

11. Centers for Disease Control and Prevention. Sexually Transmitted Disease Surveillance 2015. Atlanta: US Department of Health and Human Services; 2015.

12. Fonseca-Capdevila E, Mazaira-Fernández M. El resurgimiento de la sífilis: un problema de salud pública actual en España. Piel. 2007; 22: 370-373.

13. Baughn R, Musher D. Secondary syphilitic lesions. Clin Microbiol Rev. 2005; 1: 205-216.

14. Carrada-Bravo T. Síndromes neuropsiquiátricos causados por Treponema pallidum. Rev Chil Neuro-Psiquiat. 2015; 53: 175-186.

15. Vera I, Bermejo A, Leiro V, Parra V et al. Condilomas planos interdigitales. Dermatol Argent. 2010; 16: 199-203.

16. Pérez $S$, Sisalema V, Ferreiro M, Arévalo $C$ et al. Sífilis secundaria: formas clínicas reemergentes. Derm Venez. 2000; 38: 34-37.

17. Kazlouskaya V, Wittmann C, Tsikhanouskaya I. Pustular secondary syphilis: report of three cases and review of the literature. Int J Dermatol. 2014; 53: 428-431.

18. Piraccini B, Broccoli A, Starace M, Gaspari V et al. Hair and scalp manifestations in secondary syphilis: epidemiology, clinical features and trichoscopy. Dermatology. 2015; 231: 171-176. 